\title{
Examining mixed unknown distributions (mud)
}

\author{
DANIEL B. WRIGHT \\ University of Sussex, Brighton, England \\ and Florida International University, Miami, Florida \\ AND \\ ELIN M. SKagerberg \\ University of Sussex, Brighton, England
}

\begin{abstract}
A function, written in $\mathbf{R}$, for testing whether the distribution of responses in one condition can be considered a combination of the distributions from two other conditions is described. The important aspect of this function is that it does not make any assumptions about the shape of the distributions. It is based on the KolmogorovSmirnov $D$ statistic. The function also allows the user to test more specific and, hence, more statistically powerful hypotheses. One hypothesis, that the mixture does not capture the middle third of the distribution, is included as a built-in option, and code is provided so that other alternatives can easily be run. A power analysis reveals that the function is most likely to detect a difference between the combined conditions' distribution and the other distribution when the center of the other distribution is near the midpoint of the two original distributions. Critical $p$ values are estimated for each set of distributions, using bootstrap methods. An example from human memory research, exploring the blending hypothesis of the misinformation effect, is used for illustrative purposes.
\end{abstract}

Problem. Let Dist1 and Dist2 be two distributions of variables, without making any assumptions about their shape. Our question is whether a third distribution, Dist3, can be accounted for by a combination of Dist 1 and Dist2, so that prop Dist1 + $(1-$ prop $)$ Dist2 $=$ Dist3, where prop (short for proportion) is an unknown value between 0 and 1. The problem is to find a good value for prop and decide whether the combination of these two distributions provides an adequate fit for Dist3. There are procedures/ functions available for this if one makes assumptions about the shape of the distributions of Distl and Dist2, but not if these assumptions are not made. A function in $R$ (R Development Core Team, 2007) was developed for this situation and is described.

Example. For more than 30 years, memory researchers have been interested in what happens when people see some event and, later, have some detail of this event inaccurately described (Loftus, 2005; Wright \& Loftus, 2008). The basic finding is that some people will report the errant postevent information. This has become known as the misinformation effect. Figure 1 shows a schematic of the typical experimental protocol and two of the main explanations (Wright, 1993). To make this example concrete, we assume that, in the original presentation, the participant sees a car at a stop sign, and later it is suggested that the car was at a yield sign (Loftus, Miller, \& Burns, 1978). The first explanation is that the participant maintains intact representations for the two signs and, depending on the retrieval tasks, reports one of these. This is shown by the separate stop and yield signs in the top right-hand corner of Figure 1. The second explanation is that the person reports some blend of the 2 objects; in the lower righthand corner of Figure 1, this is depicted by a yield sign with the word STOP. This may be due to the person's having only a blend representation of the two objects or simply reporting a compromise of the two.

With stimuli like those in Figure 1, it is difficult to test for the blend explanation, because it is unclear what the blend of a stop sign and a yield sign should be. Because of this, some researchers (e.g., Belli, 1988; Loftus, 1977) have used original and postevent information that vary along a scale, so that it is easier to conceive of a blend of them. In our own research (Skagerberg \& Wright, in press), we have used colors (where participants choose from a color chart), the height and build of a person, and count variables. In the example we use for illustration, we showed people several videos, including one of a train station. We consider three groups: (1) a control group who saw 18 people at the station, (2) a control group who saw 6 people at the station, and (3) an experimental group who saw 18 people at the station but then received postevent information from somebody who saw only 6 people.

When the participants were given a memory test, the means for these three groups were $16.71,6.10$, and 12.08 people, respectively. So, the means for the two control groups were near their true values, and $t$ tests showed that the experimental group was significantly different from each of these. However, both explanations shown in Fig- 
Representations exist intact.

One or the other is retrieved.

Estimate probability of each.

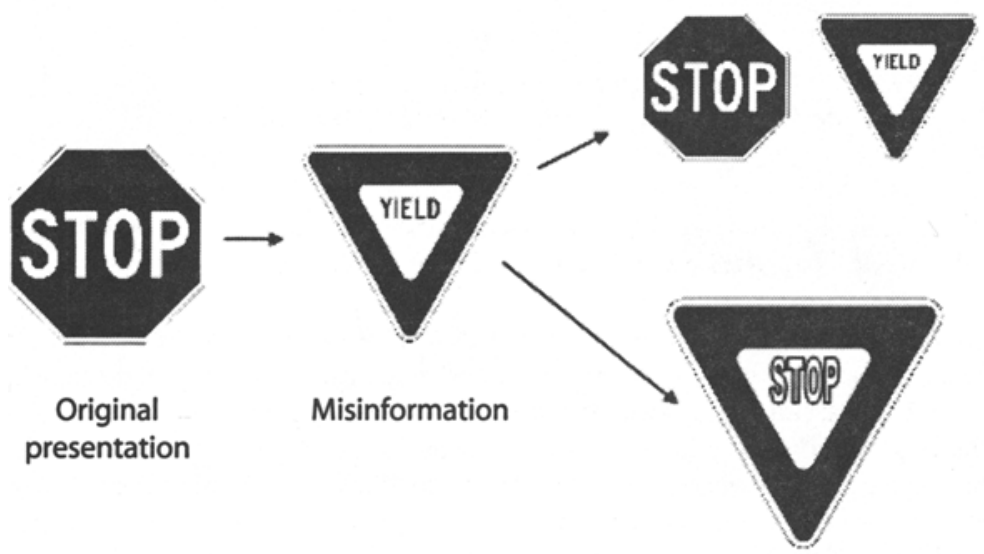

A blend of the two is retrieved. In many situations it is unclear what this blend would be.

Figure 1. The design for a postevent misinformation study.
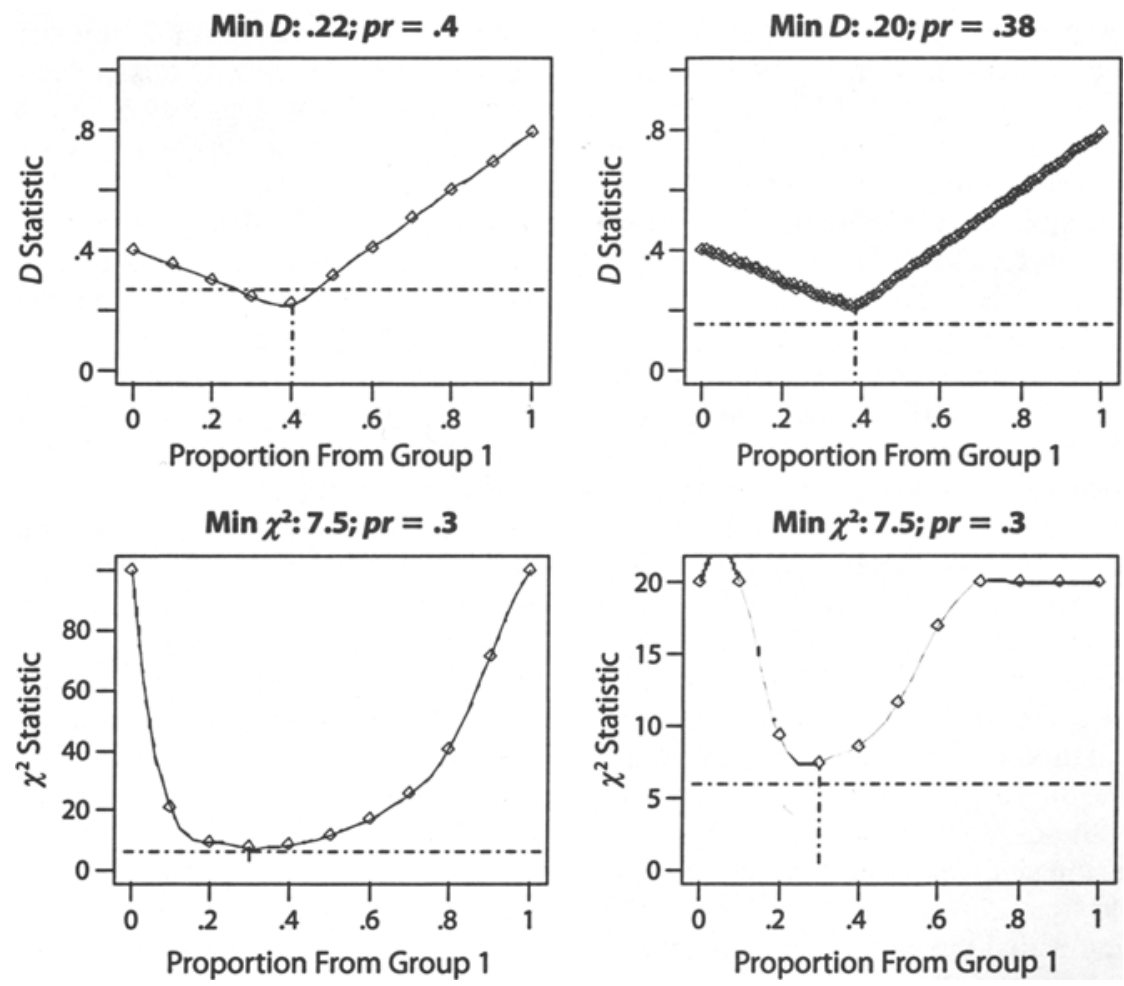

Figure 2. The output from the mud. The first graph (upper left-hand corner) shows that the minimum $D$ is where about $40 \%$ from the first control group and $60 \%$ from the second control group are used for the mixture. In the second graph (upper right-hand corner), the precision is increased, and the critical $p$ value is found through a bootstrap method (and the minimum value is now statistically significant). The third and fourth graphs (left and right panels of the bottom row) show the comparisons among thirds, a more powerful comparison. The difference between these two graphs is in the settings of chimax. 
ure 1 predict that the mean for the experimental group should be between the means for the two control groups. To differentiate these explanations, the distributions of the three groups need to be compared. The most common approach is to use the empirical distributions from the control groups to estimate population distributions and then test whether some combination of the two estimated control distributions can create the distribution observed for the experimental group. If one is willing to assume that the two control groups are drawn from populations with known distributions, existing software (e.g., mixdist; Macdonald \& Green, 1988, www.math .mcmaster.ca/peter/mix/mix.html) can be used. The problem with this approach is that testing the fit of the combined control distributions to the distribution observed in the experimental group can yield a significant result because the assumed population distributions are inadequate, rather than because the experimental group's distribution is not a weighted combination of those for the control groups. We searched for a mixture technique without these assumptions, and the function developed and reported here is such a technique.

\section{Mixing Unknown Distributions (mud)}

Most statistical work on mixture distributions is done to determine whether a single empirical distribution is composed of several known distributions. There has been rela- tively less research in which unknown distributions have been compared. Maiboroda (2000) has conducted some of the most relevant research in the Ukraine, but much of this research has not been translated. His approach is similar to the approach taken here, building on the KolmogorovSmirnov statistic, $D$.

The Kolmogorov-Smirnov statistic, $D$, is often used to compare two distributions when one is unwilling to make assumptions about the shape of the distributions. The procedure works by comparing their cumulative distributions and recording the largest difference between these two distributions (Siegel \& Castellan, 1988; Wilcox, 1997, 2005). Here, the problem is to create a mixture of two distributions, where the relative amount of each is unknown, and to compare this combined distribution with an empirical one. There are several alternatives and modifications to the basic Kolmogorov-Smirnov D (e.g., Damico, 2004). Probably the most discussed of these is the Cramér-von Mises procedure, but the research comparing this with $D$ has yielded no clear winner (Wilcox, 2005). Some adaptive forms of these tests have been proposed and can yield more powerful results (Büning, 2001), but because of its simplicity, popularity, and relatively good properties, we chose to use the Kolmogorov-Smirnov $D$ as the main test statistic.

The function, called mud (for mixing unknown distributions) takes two distributions and creates different
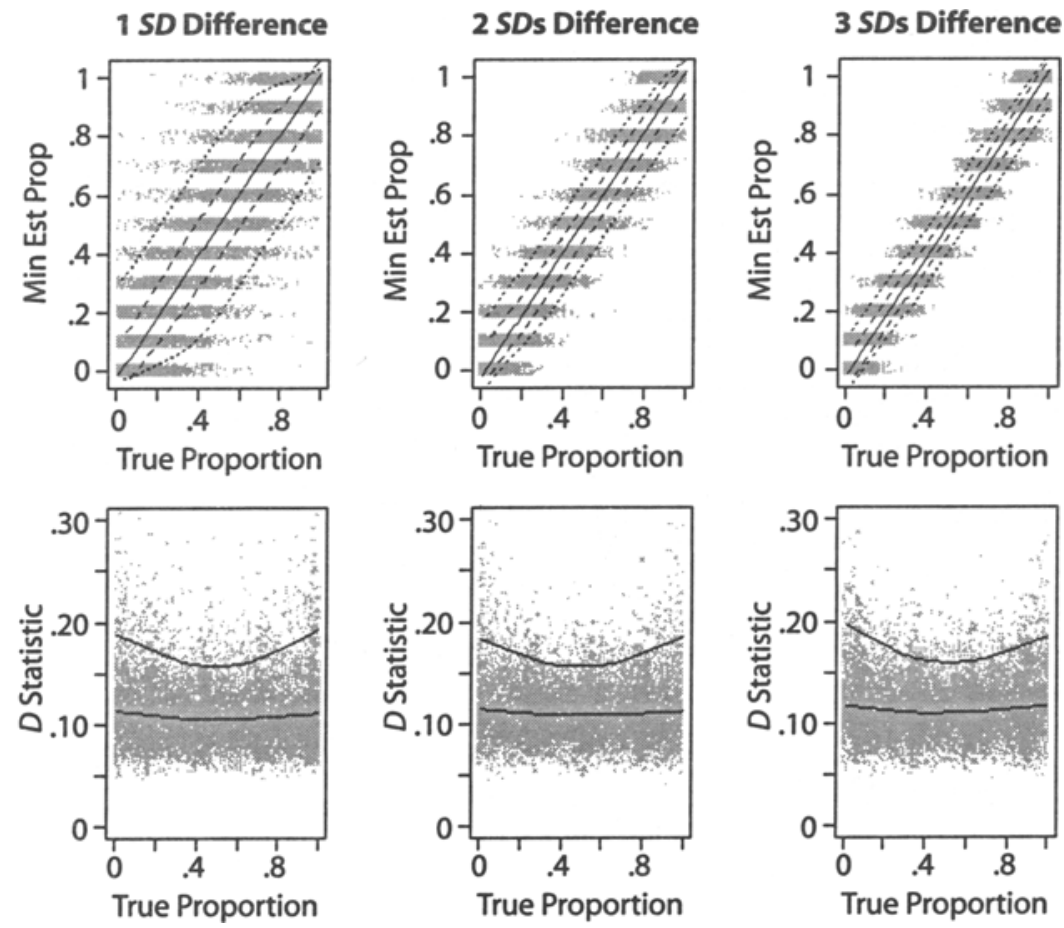

Figure 3. The performance of method=1 (Kolmogorov-Smirnov's $D$ ) of mud when the third distribution is a mixture of the first two, with $n=30$ and three effect sizes. The top row shows the estimates of the proportion. The solid lines are for the medians, the dashed lines for the 25th and 75th percentiles, and the dotted lines for the 5th and 95th percentiles. The bottom row shows the estimates of the $D$ statistics. The top lines show the 95th percentiles (corresponding to the traditional $5 \%$ significance level), and the bottom lines show the medians. 
proportions of them to produce a graph that will help the user to determine whether the third distribution might be a weighted sum of the first two. The function works by first comparing one of the original two distributions with the third and then, in steps, mixing in part of the other original distribution until the mixture is $100 \%$ of it. The number of steps is determined by a parameter, precision, so that precision $=.1$ takes 10 steps and precision $=.01$ takes 100 steps. The default is .1 .

In general, the Kolmogorov-Smirnov test can be run as either a one-sample or a two-sample test. Within R, the ks. test function allows only one-sample tests against a set of named distributions. To mimic this situation, the default for mud is to run a two-sample test, but with a very large sample for the mixture (the default is si ze $=10,000$, but this can be changed, and increasing the size does not seriously affect the speed of the processing). When one is interested in the power of tests and in rejecting/accepting hypotheses, it can be useful to set size=length (bg) and samps to a large number. This will add intervals to the graph of $D$ values by the proportion of the mixture of the two control groups (the confidence interval level can also be changed from the default $C I=.95$ ). The mean of bootstrap samples is printed, so if samps is a small number, the solution will not be reliable.

The Kolmogorov-Smirnov $D$ is sensitive to variation in the distributions throughout the distributions (although the nonweighted version of the test that we use is more sensitive in the middle [Wilcox, 2005], which is appropriate here). This is done at a cost. If there is a more specific hypothesis, $D$ has low relative power. In the example we discuss, there is interest in whether a mixture of the two control distributions captures the middle of the experimental distribution adequately. This more specific hypothesis can be examined in several ways. One method is to split the experimental condition into thirds and compare these scores with the expected probabilities from different mixtures of the two control groups, using a $\chi^{2}$ statistic with 2 degrees of freedom. This example is just a single alternative, but it seems useful enough to have a built-in function as an option (set method=2). Because the expected cell probabilities can be used in R's one-sample chisq . test function, the size parameter is not necessary. For the accompanying graphs, because $\chi^{2}$ can be infinite, there is a chimax parameter with a default of 100 , which sets all values above it to that value. Because the $\chi^{2}$ values are likely to be positively skewed, this prevents outliers (including those of $+\infty$ ) from having a large influence. In many cases, it is worth using a lower value, so that it is easier to observe at which proportion the minimum $\chi^{2}$ value occurs. Similarly, there are some situations in which it may be advisable to increase the chimax value.

By default, mud prints a horizontal dashed line for the traditional single-test critical $p$ value at $5 \%$. The approxi-
1 SD Difference
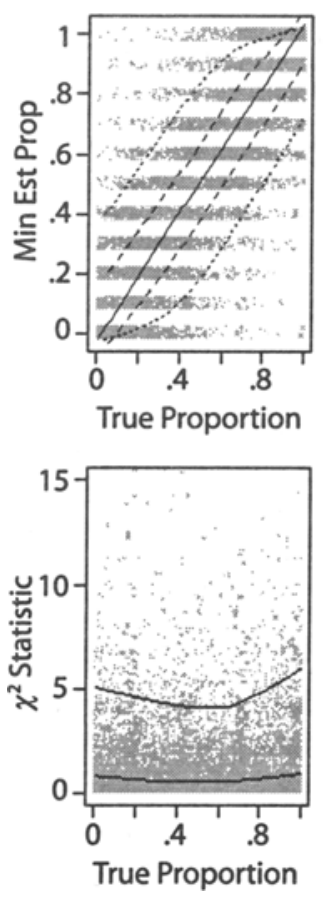

2 SDs Difference
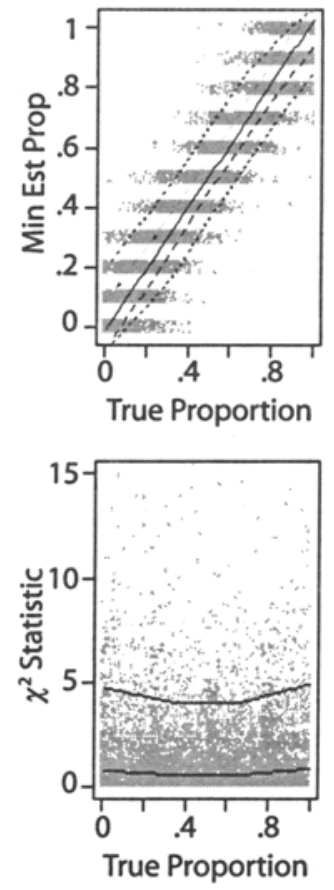

3 SDs Difference
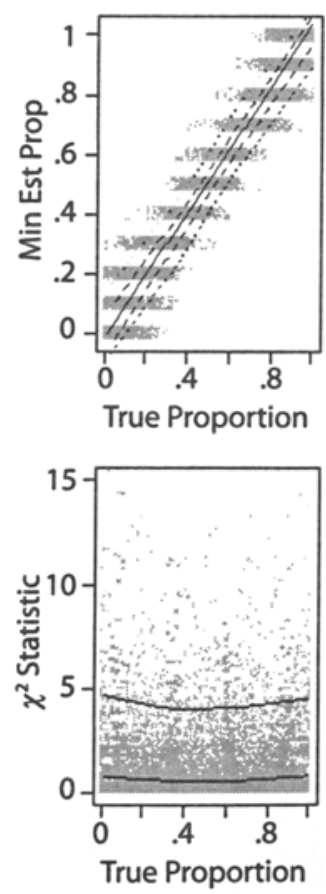

Figure 4. The performance of method $=2$ (comparing thirds with the $\chi^{2}$ statistic) of mud when the third distribution is a mixture of the first two, with $n=30$ and three effect sizes. The top row shows the estimates of the proportion. The solid lines are for the medians, the dashed lines for the 25th and 75th percentiles, and the dotted lines for the 5th and 95th percentiles. The bottom row shows the estimates of the $\boldsymbol{X}^{2}$ statisties. The top lines show the 95th percentiles (corresponding to the traditional $5 \%$ significance level), and the bottom lines show the medians. 
mate critical $p=.05$ for a single test of $D$ is $1.36 / \sqrt{n}$ (Siegel \& Castellan, 1988), and for $\chi^{2}(2)$, it is 5.99. The usual limitations of $p$ that exist with a stepwise regression, where the search choice of predictors is chosen to produce a small $p$, are present. The simulations reported below show that these traditional critical levels are quite conservative. A bootstrap estimate can be printed by letting $c r i t p=2$. Setting $c r i t p=3$ means that no horizontal line is printed.

Figure 2 shows the graphical output from the mud function with data from Skagerberg and Wright (in press) and the following commands:

$\exp 18<-c(10,8,20,15,13,15,12,8,9,10$, $15,8,7,15,6,15,20,15,11,15,6,20,15,8,6)$ control18<- $c(15,18,20,15,17,10,15,20$, $15,12,20,20,12,12,18,18,27,15,22,10,20)$ control6 <- $c(6,10,6,6,5,6,5,5,6,6,6,6$ $, 5,6,7,7,5,7,6,6,6)$

$\operatorname{par}(\operatorname{mfrow}=\mathrm{c}(2,2))$

mud (control6, control18, exp18)

mud (control6, control18, exp18, critp $=2$, precision $=.01$ )

mud (control6, control18, $\exp 18$, method=2) mud (control6, control18, exp18, method=2, chimax $=20$ )

In addition to the graphical output, mud outputs the statistic $\left(D\right.$ or $\left.\chi^{2}\right)$, the proportion from Group 1 , and the $2.5 \%$ and $97.5 \%$ percentiles (if $C I=.95$ and samps $>1$ ) for each proportion tested.

The graph in the upper left-hand corner of Figure 2 shows the default settings for mud. It shows that the best-fitting mixture has about $40 \%$ from the first control group and $60 \%$ from the second group. The minimum value is $D=.22$, which is less than is needed to reach statistical significance for the traditional method for calculating the critical $p$, and therefore, using this method, the statistic is nonsignificant. In the graph to the right, the precision is increased, and the critical $p$ is calculated using bootstrap methods. The minimum $D(.20)$ is now about the line for the new critical $p$, and therefore, the statistic is statistically significant. This means that we can reject the hypothesis that a mixture of the two control distributions can re-create the experimental group's distribution. The bottom two graphs use method $=2$ for the comparisons between thirds. They show that this method is more powerful and yields a statistically significant result (even with the traditional critical $p$ of 5.99 ; a bootstrap estimate of the critical value is 3.67). The difference between these two graphs is that chimax $=100$ (default) is used in the left panel and chimax $=20$ is used in the right panel. The latter allows the user to look more closely at where the $\chi^{2}$ is near its minimum. Text output also provides this information. The chimax option is used, rather than simply changing the $R$ graphical parameter $y \mathrm{im}$, because of the problem cited above that the $\chi^{2}$ values will usually be skewed and some values may be infinite.

\section{Evaluating the Function mad When the Distribution Is a Mixture}

Simulations were conducted to see how well mud is able to estimate the true mixture proportion from two groups to create a third. Many different shapes of distributions could have been used, but for simplicity, we used control groups that were normally distributed with equal variances and differences between them of $\sigma=1,2,3$. Because differentshaped distributions produce different results, these simulations provide a rough guide for performance. In conventional effect sizes, these are all large, but if the control groups are not easily differentiated, there is no point in trying to differentiate between hypotheses about combinations of them. For these simulations, a third variable was created to have proportions from 0 to 1.00 , in steps of .01 , from one distribution and the remainder from the other. Thus, the null hypothesis, that the third distribution is a mixture of the first two, was true. One hundred replications were done at each of the 101 steps, and test statistics were calculated. One hundred replications yielded stable enough results that more replications were not necessary. This was done for both the Kolmogorov-Smirnov $D$ and the $\chi^{2}$ methods. Different sample sizes were examined, but in this section, we will focus on $n=30$.

The top row of Figure 3 shows how well the function was able to estimate the true proportions used to create the mixture distribution. The lines through the data are robust regressions (using the lowess function in R) through the $5 \%, 25 \%, 50 \%, 75 \%$, and $95 \%$ percentiles for each of the 101 different true proportions. The median line (solid) is very close to the diagonal for all three graphs, showing that the estimates for the proportion of the mixture appear unbiased. The variability of estimates is best examined by looking at the dashed and dotted lines. The lines containing $50 \%$ of the data (the dashed lines) are relatively straight (except at the ends, where they have to curve, since the estimates are constrained between 0 and 1), and therefore, there does not seem to be a large effect of what the true proportion is on the estimate's variability. When the control distributions are one standard deviation apart, the $50 \%$ band has a width of .23. When they are two standard deviations apart, the width is .12, and when three standard deviations apart, it is .08 . Thus, as the effect size for the difference between the control groups gets larger, the accuracy for identifying the true proportion of the mixture also increases. When the sample size was increased, the width of the $50 \%$ bands decreased (e.g., for $n=100$, the respective bands for standard deviations of 1,2 , and 3 were $.14, .07$, and .04, respectively).

The second row of graphs shows all the $D$ values. The curves (again, the lowess function was used) show the median values and the 95 th percentiles. All of these graphs have a similar U-shaped pattern for the 95th percentile. This level, which corresponds to traditional significance level of $5 \%$, shows that Type I errors (i.e., $D$ values above some cutoff) are more likely when the true proportion is mostly from one of the two distributions, and not from an even mixture of the two distributions (if a single critical $D$ value is assumed). The minimum values for these three graphs are .16, .15, and .16. The traditional (asymptotic) critical value for one-sample Kolmogorov-Smirnov tests, $1.36 / \sqrt{n}$, is .25 . We reran these simulations with $n=100$, and the minimum observed values were $.09, .10$, and .10 , 

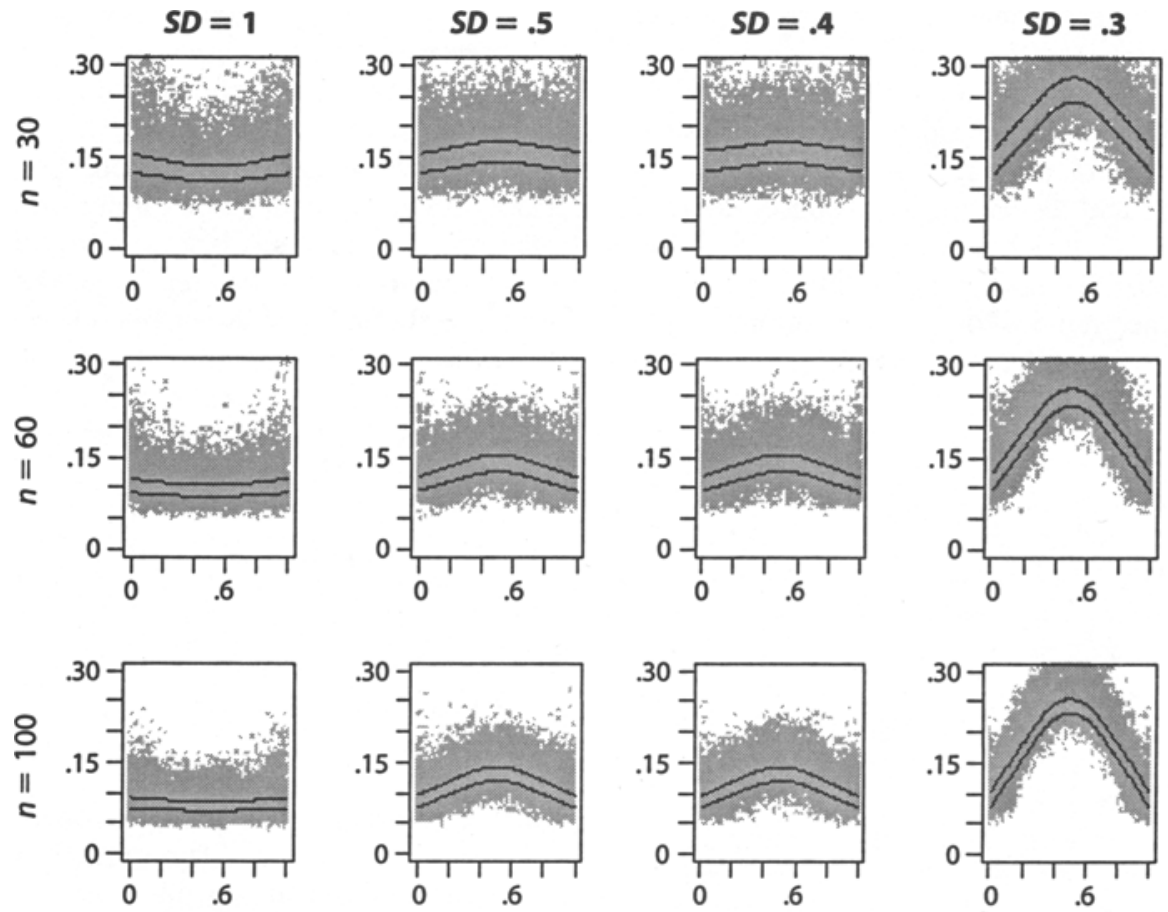

Distribution Center

Distribution Center

Distribution Center

Distribution Center

Figure 5. Detecting an effect using the Kolmogorov-Smirnov $D$ statistic method for different effect sizes and sample sizes. The lines are the 50th and 20th percentiles (lowess curves). The 20th percentile matches onto the convention that, for power, $80 \%$ of the time one should reject the false null hypothesis.
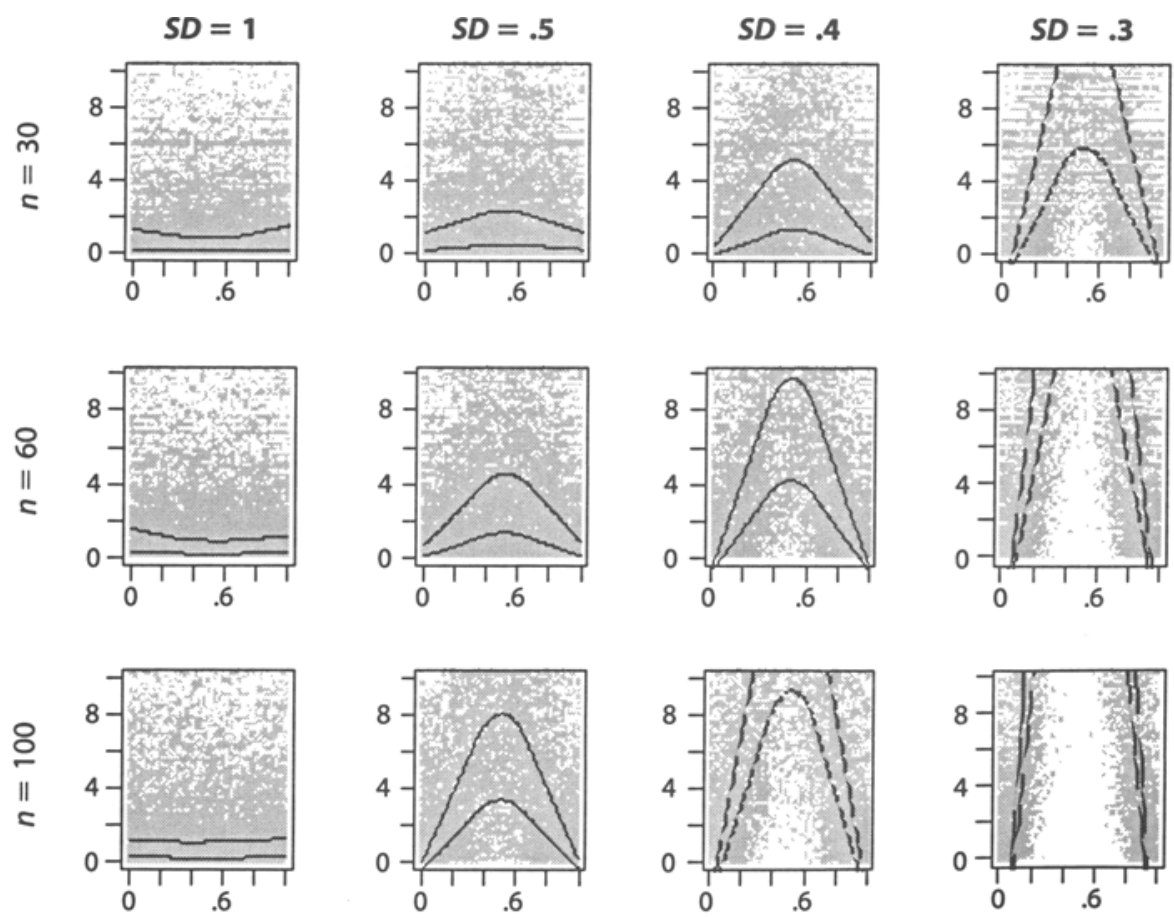

Distribution Center

Distribution Center

Distribution Center

Distribution Center

Figure 6. Detecting an effect using the comparing thirds method (method=2) for different effect sizes and sample sizes. The lines are the 50th and 20th percentiles (lowess curves). The 20th percentile matches onto the convention that, for power, $80 \%$ of the time one should reject the false null hypothesis. 

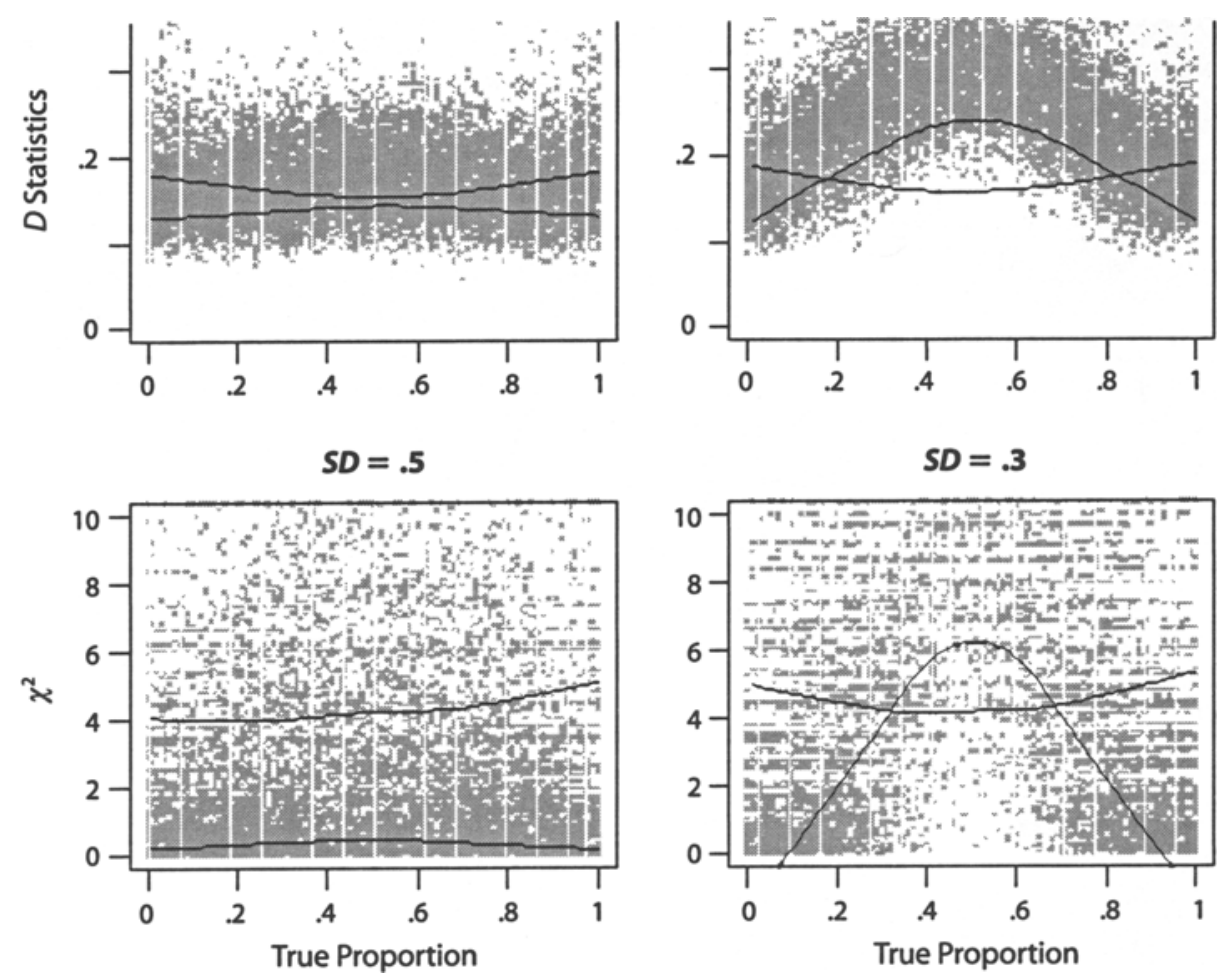

Figure 7. Comparing the 95th percentile for falsely rejecting a true mixture (the higher lines at the extremes) with the 20th percentiles for falsely not rejecting an effect. The area between the points at which these lines intersect in the right two graphs corresponds to conventional guidelines for acceptable power.

for which the traditional critical value is .14. On the basis of this, we recommend using the mudp function, described below, and the critp $=2$ option in mud for final graphs. We did not make this the default option for mud, because it is much slower than the default critp=1.

Figure 4 shows the corresponding graphs for method $=2$, when the thirds are compared using the $\chi^{2}$ test. The estimates of the true proportion are again unbiased, and as is shown by the dashed lines (enclosing 50\% of the cases), the estimates of the true proportion are approximately as accurate (the widths are $.25, .09$, and .08 ) as with the Kolmogorov-Smirnov method. The bottom row of graphs also shows the U-shaped pattern evident in Figure 3, but it is less pronounced. The minimum $\chi^{2}$ values for the 95th percentile were $4.05,3.96$, and 4.00. The critical $\chi^{2}$ value for two degrees of freedom is 5.99; so, as with the $D$ statistic, the estimates are conservative.

\section{Evaluating the Function mud When the Distribution Is Not a Mixture}

Next, we examined how well mud could correctly identify that a distribution was not a mixture of those for the two control groups. The simulations were run so that the means for the control groups were 1 unit apart and so that the mean for the third distribution ranged between them in steps of .01 . Four different standard deviations were used: $1, .5, .4$, and .3 . Figures 5 and 6 show the graphs for $D$ and $\chi^{2}$, respectively. We printed three sample sizes: $n=30,60$, and 100 . The lowess curves correspond to the 50 th percentile (the median) and the 20th percentile. The 20 th percentile was chosen because it corresponds to the conventional rule of thumb that power should be approximately $80 \%$ (Baguley, 2004).

Both Figures 5 and 6 show a similar pattern. As the effect size increases (going from left to right) and the curves within the individual graphs move up, and as the effect size gets larger (for $S D=.3$, and the pattern continues for larger effects not shown in this figure), the lines arc upward in the middle. As the sample size increases from the first row $(n=30)$ to the final row $(n=$ 100 ), two things are most notable. First, for the $D$ statistic graphs, the median lines do not change that much, but for the $\chi^{2}$ graphs, they do, because the former is a measure of effect size and the latter is not. Second, the lines become closer as the sample size increases. This shows that the power is increasing. Eighty percent of the values are above the lower line (the 20th percentile), and as this line moves up, the likelihood of observing a statistically significant result also increases (i.e., the power increases). In comparing Figures 5 and 6 , although the lines for the median curve begin to arc more quickly as the effect and sample size increase for those graphs in 


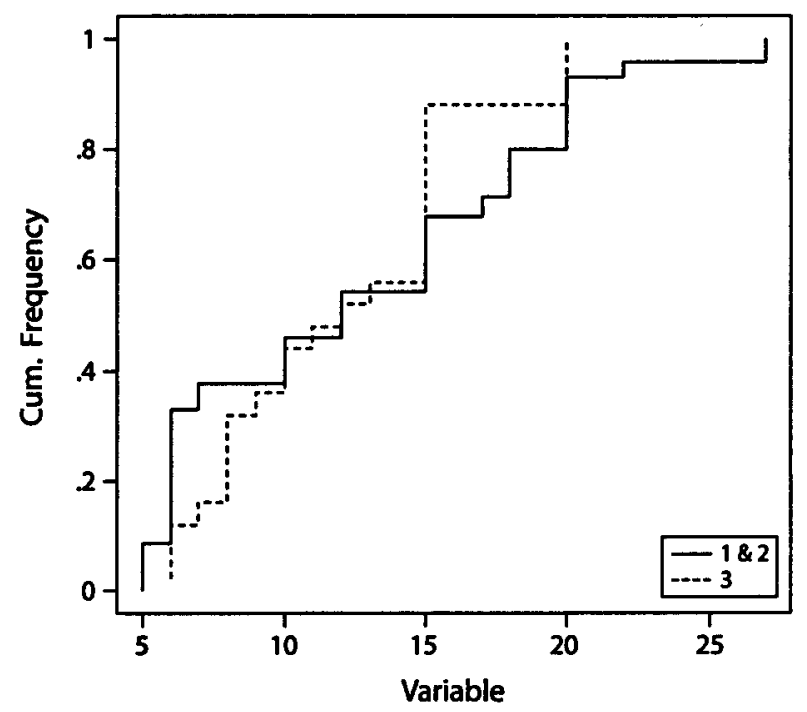

Figure 8. Comparison of the cumulative distributions for a mixture of the two control conditions and the third condition, using the mudsolution function.
Figure 6 than for those in Figure 5, the differences are less pronounced for the 20th percentile lines.

Figure 7 compares the 95th percentile lines from Figures 3 and 4 with the 20th percentile lines from Figures 5 and 6 , for $n=30$ and two effect sizes. With this small sample size, it is clear for $S D=.5$ that neither method reaches the conventional level of power, where these lines overlap. As the effect size is increased, both reach the conventional levels when the third distribution has a mean about halfway between those for the two control groups. As the sample size is increased, both these methods increase in power.

\section{The mudp and mudsolution Functions}

Two additional functions were written: mudp and mudsolution. The simulations above show that for normal distributions, the mud function provides good estimates for the proportion but that the test statistics are conservative. How well the function performs is dependent on the distributions. Although the simulations show that critical test statistics could be used that are lower than the
Control 1

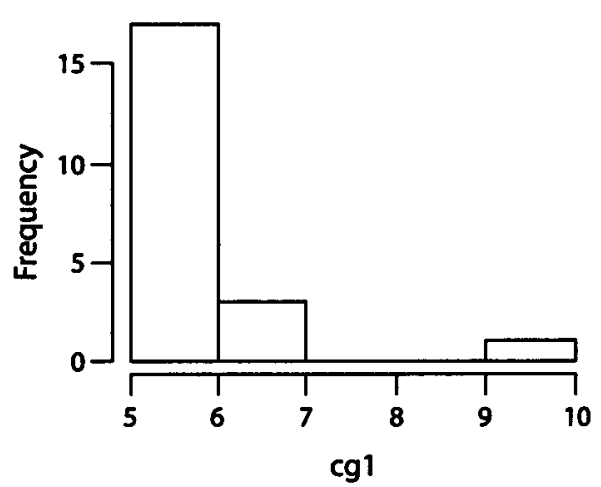

Blended

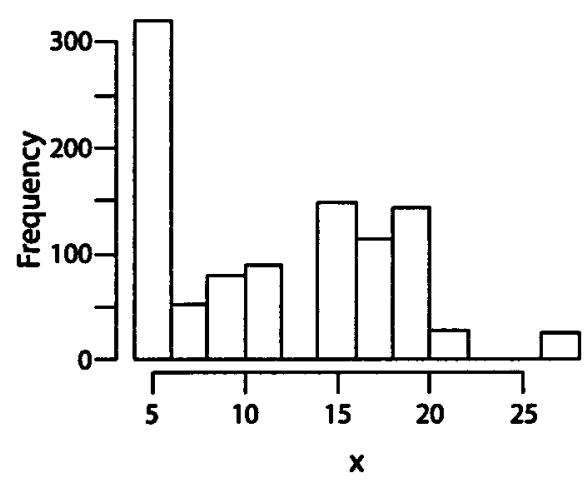

Control 2

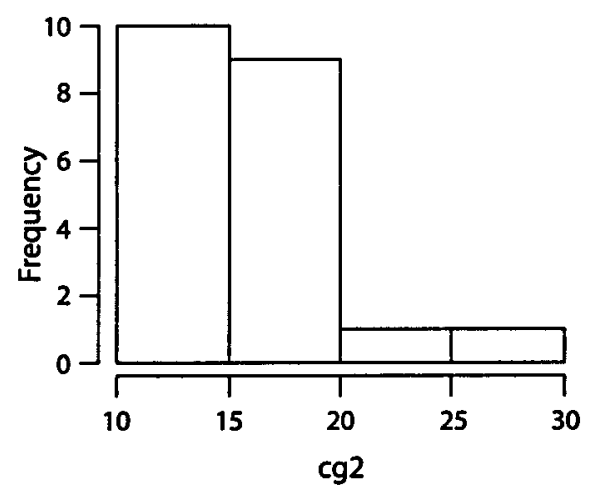

Experimental

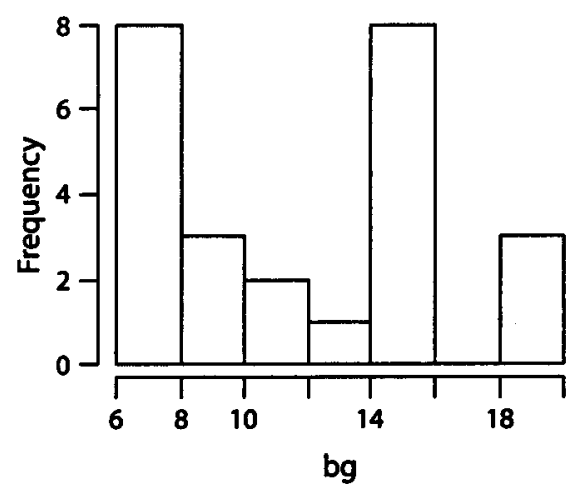

Figure 9. The four histograms that are plotted by mudsolution when hist=T. These are the histograms for the two distributions to- be- blended (top row), the blended distribution based on the proportion entered into the function (lower left-hand corner; the frequency on the $y$-axis can be varied with the size parameter), and the observed distribution with which this blend is compared (lower right-hand comer). 
traditional values for $D$ and $\chi^{2}$, the values obtained from the normal distribution simulations should not be used to provide estimates for the $p$ values.

The purpose of mudp is to provide better estimates of the critical $p$ values, using a bootstrap procedure. To calculate these, mudp takes the two control distributions and combines them on the basis of the proportion found with mud (or any other proportion entered with the prop parameter) and calculates either the minimum $D$ (method $=1$ ) or $\chi^{2}$ (method $=2$ ), using a slimmed down version of mud called mud2. The default is for this to be done 1,000 times, but the number can be controlled with the replicat parameter. The estimated critical $p$ values for $10 \%, 5 \%, 2 \%$, and $1 \%$ are reported. For Skagerberg and Wright's (in press) data, the following commands yield these critical $p$ values (the critical values for the $5 \%$ level are shown in boldface here):

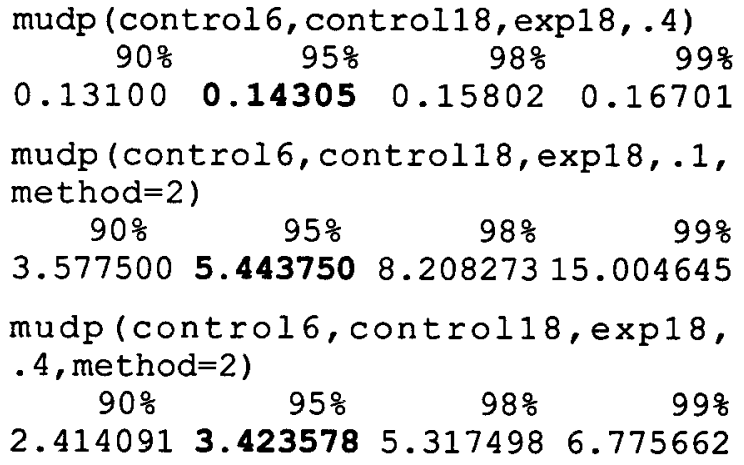

The approximation usually used for the critical $D$ at the $5 \%$ level is $1.36 / \sqrt{n}$, which, with $n=25$ for $\exp 18$, is .27. This is higher than that shown with the bootstrap estimate in mudp, and this is generally what we find. mudp also produces a critical value for the $\chi^{2}$ version of mud that is lower than the critical value for its distribution with $d f=2$, which is 5.99 . The difference between the critical values for the final two mudp functions, where prop changes from .1 to .4 , reflects the curvilinear patterns shown in Figures 3 and 4.

The Kolmogorov-Smirnov method provides no information about where the mixture distribution fails to capture the observed distribution. The mudsolution function plots the cumulative empirical distribution function with the mixture distribution produced by mud. Other than the three variables, this function includes the size parameter, which has the same meaning and default as within mud. The command mudsolution (control 6 , control18, exp 18, .4) produces Figure 8 and also outputs the $D$ statistic. This function uses the ecdf function from the Hmi sc library (Harrell et al., 2007), not the ecdf function in stats, so the Hmisc library must be attached.

The mudsolution function will also print the histograms for the two control groups, the mixture of them, and the third distribution if $\mathrm{hist}=\mathrm{T}$. These are printed in a $2 \times 2$ grid (see Figure 9) after the cumulative empirical distribution graph so, if one wants to see both and, generally, the cumulative graph is preferred, one should run the function twice.

\section{AUTHOR NOTE}

Correspondence concerning this article should be addressed to D. B. Wright, Department of Psychology, University of Sussex, Brighton BN1 9QH, England (e-mail: danw@sussex.ac.uk).

\section{REFERENCES}

BaGULEY, T. (2004). Understanding statistical power in the context of applied research. Applied Ergonomics, 35, 73-80.

BELLI, R. F. (1988). Color blend retrievals: Compromise memories or deliberate compromise responses. Memory \& Cognition, 16, 314-326.

BüNING, H. (2001). Kolmogorov-Smirnov- and Cramèr-von Mises type two-sample tests with various weight functions. Communications in Statistics: Computation \& Simulation, 30, 847-865.

Damico, J. (2004). A new one-sample test for goodness-of-fit. Communications in Statistics: Theory \& Methods, 33, 181-193.

HARRELl, F. E., JR., ET AL. (2007). The Hmisc Package 3.4-3. Retrieved January 23, 2008, from cran.r-project.org/src/contrib/Descriptions/ Hmisc.html.

LoFTUS, E. F. (1977). Shifting human color memory. Memory \& Cognition, 5, 696-699.

LofTUS, E. F. (2005). Planting misinformation in the human mind: A 30-year investigation into the malleability of memory. Learning \& Memory, 12, 361-366.

LOFTUS, E. F., MiLLER, D. G., \& BuRNs, H. J. (1978). Semantic integration of verbal information into a visual memory. Journal of Experimental Psychology: Human Learning \& Memory, 4, 19-31.

MACDONALD, P. D. M., \& GREEN, P. E. J. (1988). User's guide to program MIX 2.1: An interactive program for fitting mixtures of distributions. Ontario, Canada: Ichthus Data Systems.

MAIBORODA, R. E. (2000). An asymptotically effective estimate of a distribution from a sample with varying mixture. Probability Theory \& Mathematical Statistics, 61, 121-130.

R Development CORE TEAM (2007). $R$ : A language and envimnment for statistical computing. Vienna: R Foundation for Statistical Computing. Retrieved January 23, 2008, from www.R-project.org.

SIEGEL, S., \& CASTELlAN, N. J., JR. (1988). Nonparametric statistics for the behavioral sciences (2nd ed.). New York: McGraw-Hill.

SKagerberg, E. M., \& Wright, D. B. (in press). The co-witness misinformation effect: Memory blends or memory conformity? Memory.

WiLcox, R. R. (1997). Some practical reasons for reconsidering the Kolmogorov-Smirnov test. British Journal of Mathematical \& Statistical Psychology, 50, 9-20.

WiLcox, R. R. (2005). Introduction to robust estimation and hypothesis testing (2nd ed.). San Diego: Academic Press.

WRIGHT, D. B. (1993). Misinformation and warnings in eyewitness testimony: A new testing procedure to differentiate explanations. Memory, 1, 153-166.

WRIGHT, D. B., \& LofTUs, E. F. (2008). Eyewitness memory. In G. Cohen \& M. A. Conway (Eds.), Memory in the real world (3rd ed., pp. 91-106). Hove, U.K.: Psychology Press. 


\section{APPENDIX \\ mud and Related Functions}

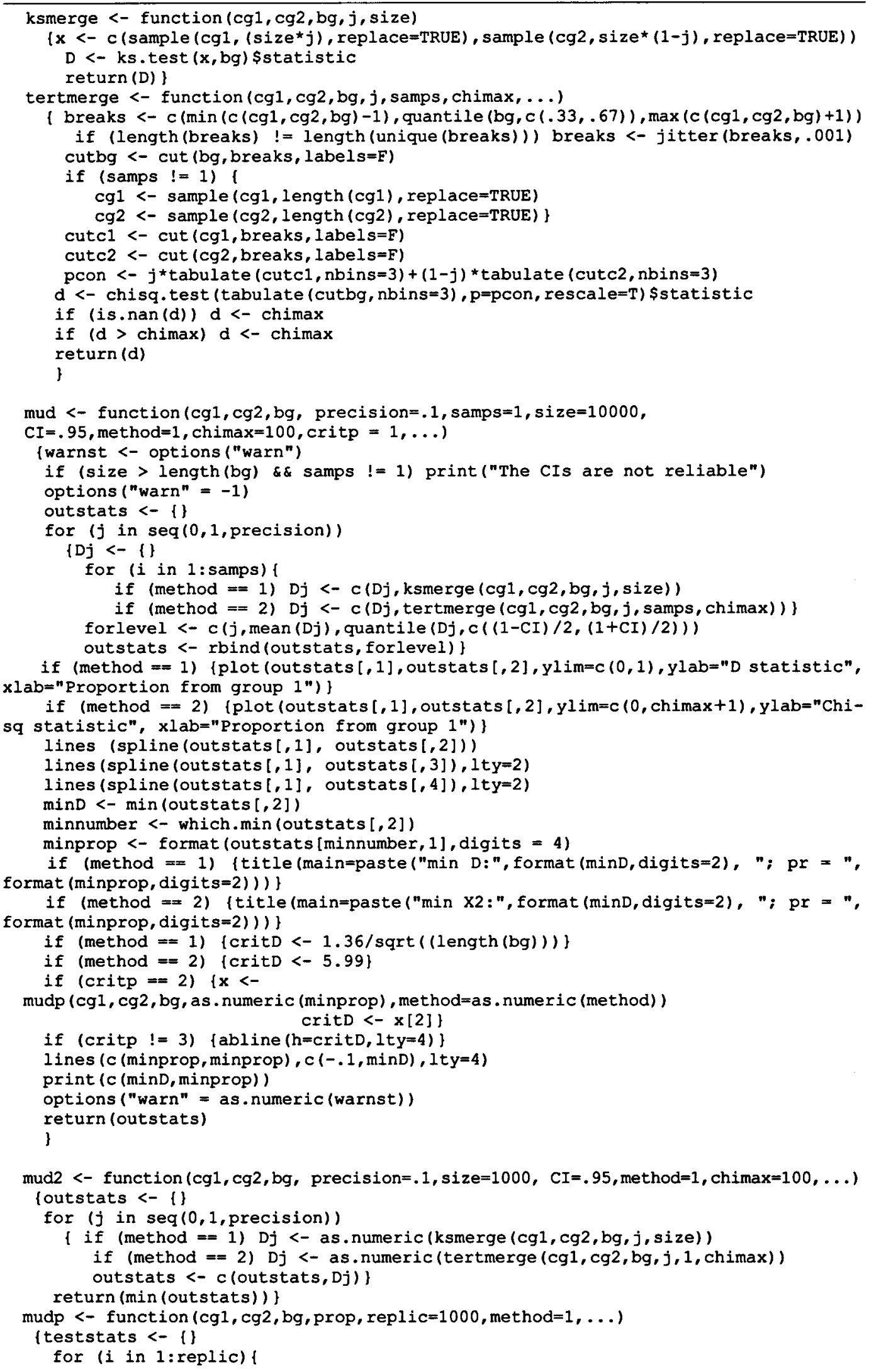




\section{APPENDIX (Continued)}

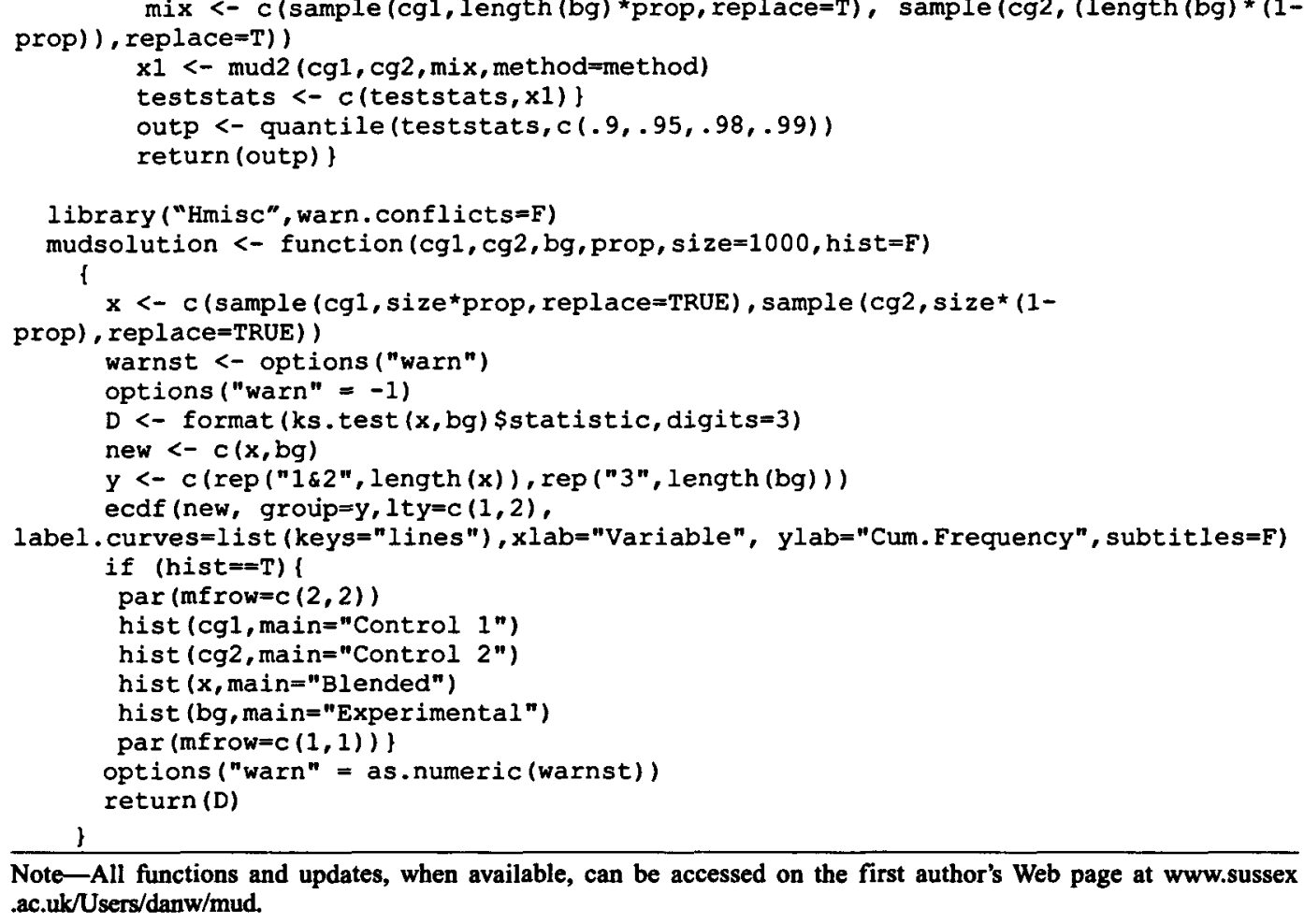

Note-All functions and updates, when available, can be accessed on the first author's Web page at www.sussex .ac.uk/Users/danw/mud.

(Manuscript received July 5, 2006;

revision accepted for publication April 2, 2007.) 